\title{
LA-UR-18-28231
}

Approved for public release; distribution is unlimited.

Title: $\quad$ DNDO Report: Developing a Photon Clutter Scaling Algorithm for Implementation into the NINESIM Tool

Author(s):

Gerez, Kyzer

McMath, Garrett Earl

Mckinney, Gregg Walter

Intended for: $\quad$ Report

Issued:

2018-08-28 
Disclaimer:

Los Alamos National Laboratory, an affirmative action/equal opportunity employer, is operated by the Los Alamos National Security, LLC for the National Nuclear Security Administration of the U.S. Department of Energy under contract DE-AC52-06NA25396. By approving this article, the publisher recognizes that the U.S. Government retains nonexclusive, royalty-free license to publish or reproduce the published form of this contribution, or to allow others to do so, for U.S. Government purposes. Los Alamos National Laboratory requests that the publisher identify this article as work performed under the auspices of the U.S. Department of Energy. Los Alamos National Laboratory strongly supports academic freedom and a researcher's right to publish; as an institution, however, the Laboratory does not endorse the viewpoint of a publication or guarantee its technical correctness. 


\title{
DNDO Report: Developing a Photon Clutter Scaling
} Algorithm for Implementation into the NINESIM Tool

\author{
Name: Kyzer Gerez \\ Hosting Site: Los Alamos National Laboratory \\ Mentors: Garrett McMath and Gregg McKinney
}

Mentor's Signature: 


\begin{abstract}
The Nuclear Inspection Node Event SIMulator (NINESIM) is a tool that is capable of modeling a node that monitors passing traffic for radiological and nuclear materials. This task requires the consideration of a number of factors. One such factor is clutter, or the level of traffic passing through a node. Clutter might affect the performance of radiation detectors due to attenuation or scattering that particles undergo as they move through the material. This paper discusses the results from MCNP simulations that were performed to determine in what scenarios clutter had a significant impact on detector performance, where the receiver operating characteristic (ROC) curve was used as the metric to evaluate detector performance. Clutter was found to have an insignificant impact on neutron detection, therefore, clutter may be ignored when modeling neutron detectors. However, there were a number of scenarios where clutter was found to affect the performance of photon detectors; the amount of traffic was found to be directly proportional to the effectiveness of photon detectors. The results of this work were then used to develop a photon clutter scaling algorithm that will be implemented into NINESIM. The Domestic Nuclear Detection Office, and possibly other agencies such as the Defense Threat Reduction Agency, will benefit from this work by eventually obtaining a tool that can enhance their ability in countering the illicit movement of radiological and nuclear materials.
\end{abstract}




\section{DNDO Report: Developing a Photon Clutter Algorithm for Implmentation into the NINESIM Tool}

\section{INTRODUCTION}

Nuclear terrorism is one of the most significant national security concerns that exist. In order to combat this threat, the United States established the Domestic Nuclear Detection Office (DNDO) within the U.S. Department of Homeland Security. This office is tasked with overseeing and improving the Global Nuclear Detection Architecture (GNDA), which is a comprehensive system with the express purpose of detecting the illicit movement of nuclear and radiological material. The GNDA is composed of nodes, each of which is a complicated, interconnected logistical system of capabilities. In order to optimize the design of an arbitrary GNDA node, the DNDO is funding a project called the Nuclear Inspection Node Event SIMulator (NINESIM).

\section{DESCRIPTION OF THE RESEARCH PROJECT}

The goal of the NINESIM project is to develop a discrete event simulation tool that can model the movement of traffic through an arbitrary GNDA node. A few factors that the tool considers include geographic location, quality of personnel, and the type of radiation detector deployed. A user can then adjust parameters to determine the optimal design of a given node.

A specific factor that must be considered when developing NINESIM is the effect of clutter on radiation detection. The clutter, or level of traffic passing through a node, might affect the ability of a radiation detector to detect photons and neutrons emitted by sources. Neglecting this scaling factor could lead to poor predictions of detector performance in certain scenarios. A modified version of MCNP6 that had a memory reduction capability was used 
in this work since the simulations required large amounts of memory [1]. The metric that was used to determine the effect of clutter on detector performance is the receiver operating characteristic (ROC) curve. The ROC curve accomplishes this task by comparing the signal plus background cumulative distribution function (CDF) to the background CDF where the former represents the probability of detection (PD) and the latter represents the probability of false alarm (PFA). For a more detailed explanation of the ROC curve and how the curve is produced in MCNP please refer to the paper titled, "How To Use The Receiver Operating Characteristic Tally Option in MCNP6" $[2,3]$.

\section{Description of the Actual Work}

\section{Simulation Details}

The GNDA node modeled consisted of five lanes with a source located on the middle lane. The source was a sphere of high-enriched uranium (HEU) based off the Godiva sphere isotopics, and this sphere was shielded by water, iron, or lead. A sodium iodide (NaI) detector was placed directly next to the source, and the detector was modeled off a design from the Scintillation Spectrometry: Gamma-Ray Spectrum Catalogue [4]. Background radiation from cosmic and terrestrial sources was enabled in the problem in order to generate the ROC curve. Clutter was approximated as a single homogeneous material that filled each lane, and this material was based off the typical composition of a car. The density of this material was then adjusted to represent different levels of traffic.

A database of results from a number of MCNP simulations with varying parameters based off the node design described was created. The parameters that were varied are listed in table 1. 
Table 1: Parameters varied in the MCNP simulations.

\begin{tabular}{lccccc}
\hline Parameters & \multicolumn{5}{c}{ Values } \\
\hline Mass $[\mathrm{kg}]$ & 5 & 10 & 20 & 30 & \\
Distance $[\mathrm{cm}]$ & 50 & 100 & 200 & 300 & 400 \\
Thickness $\left[\mathrm{g} / \mathrm{cm}^{2}\right]$ & 1 & 5 & 10 & & \\
Shield & $\mathrm{H} 2 \mathrm{O}$ & $\mathrm{Fe}$ & $\mathrm{Pb}$ & & \\
Detector Type & ${ }^{3} \mathrm{He}$ & $\mathrm{Nal}$ & & & \\
SNM Type & $\mathrm{HEU}$ & & & & \\
Clutter (\%) & 0 & 25 & 50 & 75 & 100 \\
\hline
\end{tabular}

\section{ROC Curves}

For the sake of reducing the runtime of the problems, only enough particle histories were run to allow for the mean of the pulse-height (F8) tally to converge. The ROC curve can then be generated using the calculated means during post-processing of the results. First, the probability density functions (PDF) of the signal plus background and background alone was calculated using equation (1) where $\lambda$ is the mean and $k$ is the number of counts over a selected time period.

$$
p(\lambda, k)=\frac{\lambda^{k}}{k !} e^{-\lambda}
$$

The CDF was then constructed from the PDF using equation (2).

$$
P(\lambda, k)=\int p(\lambda, k) d k
$$

Finally, the PD for a given PFA was calculated from the CDFs using equation (3) where $L_{C}$ is the detection threshold count associated with the selected PFA.

$$
P D=\int_{L_{C}}^{\infty} p(\lambda, k) d k=e^{-\lambda} \sum_{i=L_{C}}^{k_{\max }} \frac{\lambda^{i}}{i !}
$$

For each case simulated, the signal plus background CDF was plotted against the background $\mathrm{CDF}$ where the former represents the probability of detection (PD) while the latter represents the probability of false alarm (PFA). Cases with identical parameters, except for 
clutter, were grouped together, and the ROC curves of the cases in the same group were compared against each other in order to determine if clutter had a measurable impact on detector performance for the given scenario. The photon clutter scaling algorithm was then developed based off these sets of ROC curves.

\section{Results}

\section{Neutron Detection}

Figures $1-3$ show the ROC curves for the cases that included a ${ }^{3} \mathrm{He}$ detector. These figures show that clutter has a minimal effect on neutron detection, even when the problem parameters are set to maximize the effect of clutter on detector performance. Because of this, a neutron clutter scaling algorithm was deemed unneccesary for implementation into NINESIM.

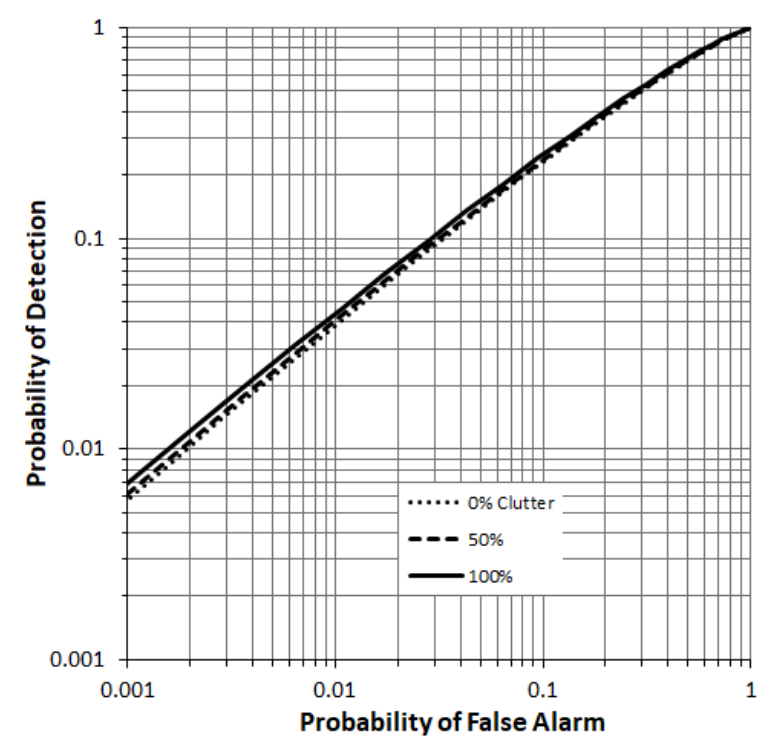

Figure 1: ROC curves of a $10 \mathrm{~kg}$ HEU sphere surrounded by lead shielding with a thickness of $10 \mathrm{~g} / \mathrm{cm}^{2}$ for a detector distance of $50 \mathrm{~cm}$.

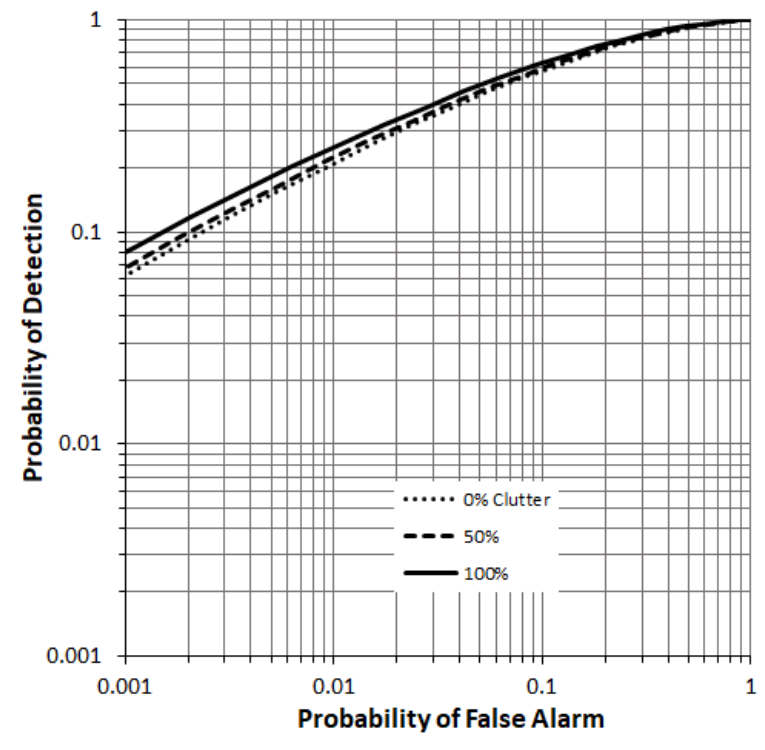

Figure 2: ROC curves of a $20 \mathrm{~kg}$ HEU sphere surrounded by lead shielding with a thickness of $10 \mathrm{~g} / \mathrm{cm}^{2}$ for a detector distance of $50 \mathrm{~cm}$. 


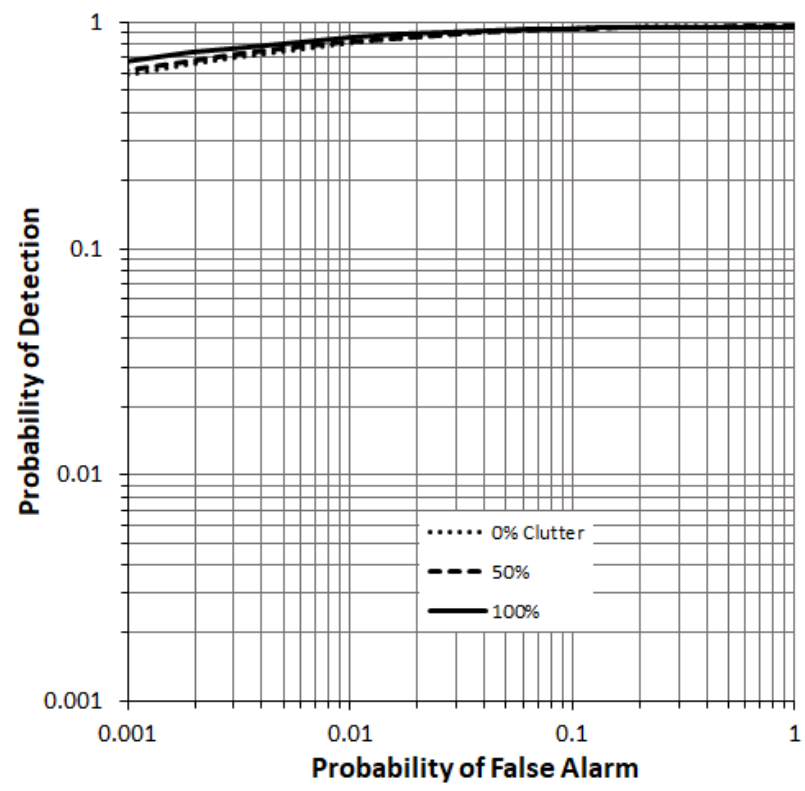

Figure 3: ROC curves of a $30 \mathrm{~kg}$ HEU sphere surrounded by lead shielding with a thickness of $10 \mathrm{~g} / \mathrm{cm}^{2}$ for a detector distance of $50 \mathrm{~cm}$.

\section{Photon Detection}

Figures $4-7$ display the photon detection results. For the photon detection results, only the PDs for a PFA of $1 \%$ are shown, not the ROC curves for each case. Due to the high number of datasets, it is not possible to show both the ROC curves and the PDs at a selected PFA for all cases. Only the latter will be displayed in order to better illustrate how PD changes as a function of clutter, as well as to show the fitting functions used. A PFA of $1 \%$ was chosen because enabling detectors to operate at a higher PFA would likely have a significant, adverse effect on commerce. In general, the graphs show that PD is significantly influenced from 0 to 50 percent clutter, with the impact of clutter diminishing when it is above 50 percent. Additionally, it is likely that some cases would not require a scaling factor due to similarity in the results. An example would be the $30 \mathrm{~kg}$ HEU sphere surrounded by lead shielding for a detector distance of $200 \mathrm{~cm}$ shown in figure 4 . However, the error of the results is unknown. An uncertainty analysis has not been performed on the ROC curves; the analysis will be carried out in future work. 


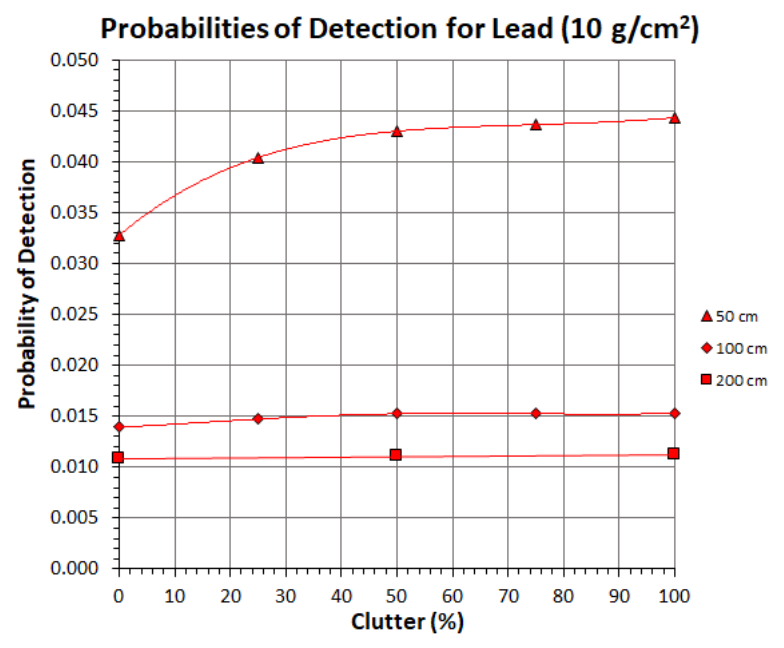

Figure 4: Probabilities of detection at a probability of false alarm of $1 \%$ for a 30 kg HEU sphere surrounded by lead shielding with a thickness of $10 \mathrm{~g} / \mathrm{cm}^{2}$.

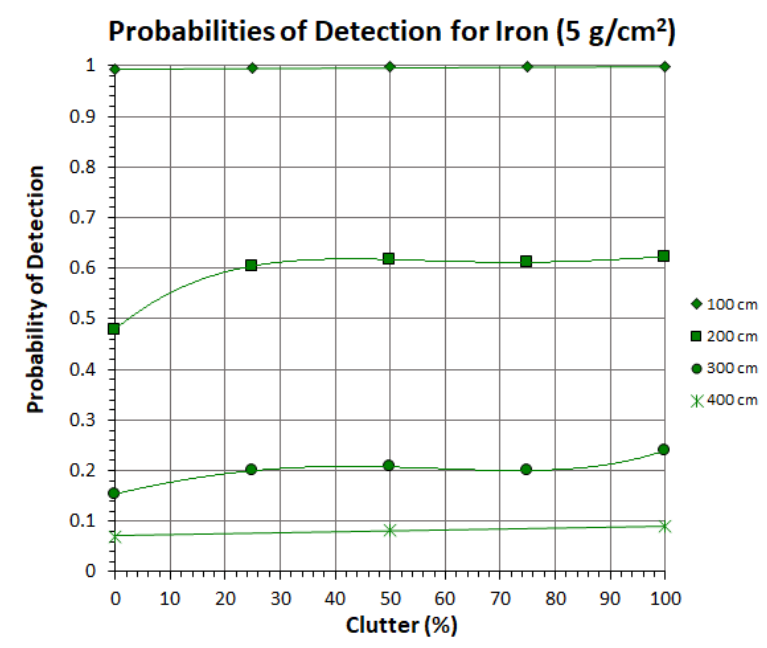

Figure 6: Probabilities of detection at a probability of false alarm of $1 \%$ for a $5 \mathrm{~kg}$ HEU sphere surrounded by lead shielding with a thickness of $5 \mathrm{~g} / \mathrm{cm}^{2}$.

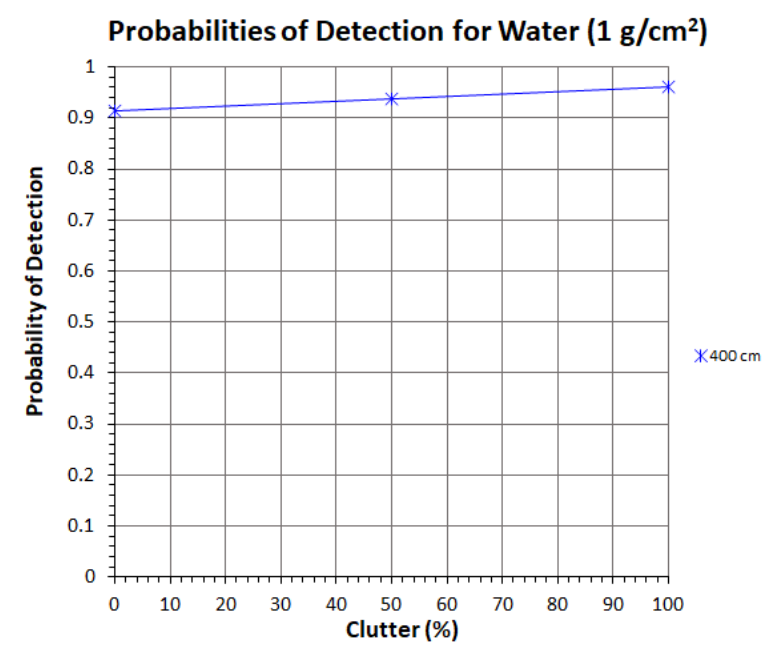

Figure 5: Probabilities of detection at a probability of false alarm of $1 \%$ for a 30 kg HEU sphere surrounded by lead shielding with a thickness of $1 \mathrm{~g} / \mathrm{cm}^{2}$.

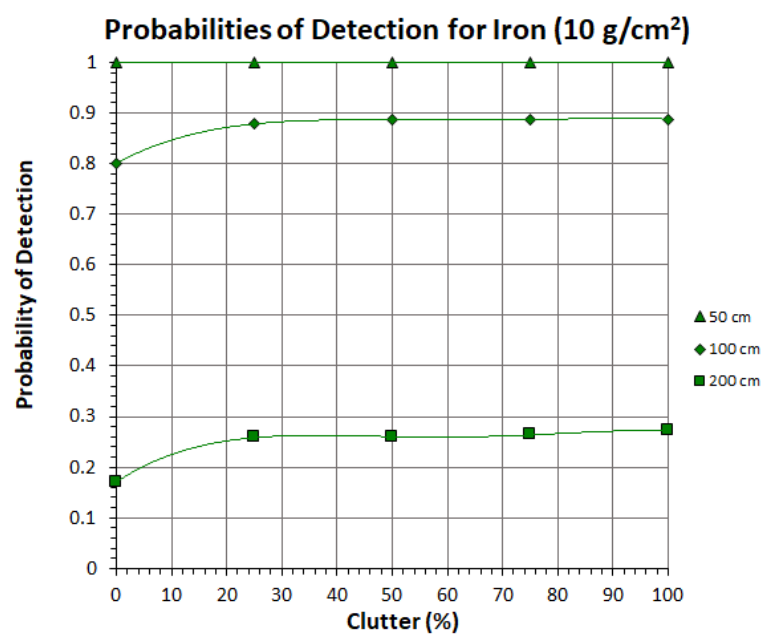

Figure 7: Probabilities of detection at a probability of false alarm of $1 \%$ for a $5 \mathrm{~kg}$ HEU sphere surrounded by iron shielding with a thickness of $10 \mathrm{~g} / \mathrm{cm}^{2}$.

The chosen fitting function for most of the data sets was a polynomial of n-1 degree where $\mathrm{n}$ is the number of data points. This was selected rather than a quadratic polynomial due to the behaviour of the datasets. In figure 7, the datasets for detector distances of $100 \mathrm{~cm}$ and 
$200 \mathrm{~cm}$ seems to behave as if they are approaching horizontal asymtopes. In figure 6, the data series for a distance of $300 \mathrm{~cm}$ experiences a "dip" at 0 percent clutter and a "rise" at 100 percent clutter. For these reasons, a quadratic polynomial seems to be an inappropriate choice for capturing the behaviour of the data. As for the rest of the datasets, a simple linear fitting function was chosen.

Figure 8 better illustrates how clutter affects photon detection. This graph shows that there were significant differences in the calculated PDs between the cases that contained clutter and the cases where clutter was excluded; these results justified the need for a photon clutter scaling algorithm. However, the graph also shows scenarios where clutter had a minimal impact on photon detection, and a clutter scaling factor may not be used in these scenarios.

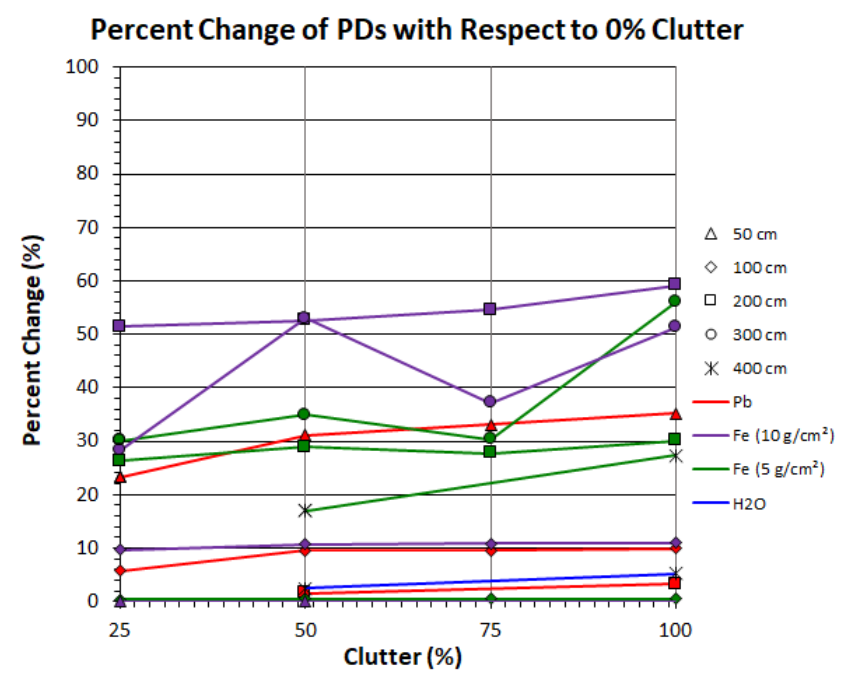

Figure 8: Percent change of the PDs for the clutter cases with respect to the PDs of cases without clutter.

In all cases, the PD appears to be proportional to the amount of clutter. Initially, these results seem unintuitive since the effect of photon attenuation is magnified as clutter increases, and the increased photon attenuation would be expected to lower the PD. However, there is a possibility that the clutter was acting as a neutron reflector, which implies that the effective neutron mutiplication factor $\left(k_{e f f}\right)$ of the HEU sphere is dependent on the amount of clutter. A number of kcode cases were simulated, and the parameters of the input decks 
are as follows: a $30 \mathrm{~kg}$ HEU sphere surrounded by lead shielding with a thickness of 10 $\mathrm{g} / \mathrm{cm}^{2}$ for a detector distance of $50 \mathrm{~cm}$. These cases either had a clutter of $0,25,50,75$, or 100 percent. Table 2 lists the results of these cases, and the results show that clutter had a negligable impact on the $k_{e f f}$.

Table 2: Effect of clutter on the $k_{e f f}$ for cases with similar parameters.

\begin{tabular}{ccc}
\hline Clutter (\%) & $\mathrm{k}_{\text {eff }}$ & Standard Deviation \\
\hline 0 & 0.87775 & 0.00072 \\
25 & 0.87746 & 0.00070 \\
50 & 0.87738 & 0.00078 \\
75 & 0.87770 & 0.00070 \\
100 & 0.87660 & 0.00079 \\
\hline
\end{tabular}

\section{Photon Clutter Scaling Algorithm}

Based off the results of the MCNP simulations, the following algorithm was developed for creating a script to adjust the PD as a function of clutter. First, the script retreives the problem parameters defined in the NINESIM input file. Then, it determines if clutter data exists for the defined problem. If this data exists, the script inputs the amount of clutter into a fitting function associated with the defined problem. The function then returns a clutter scaling factor, which adjusts the PD produced by NINESIM. If clutter data cannot be found, the script passes a scaling factor of 0.0 to NINESIM, which conveys that clutter had an insignificant effect on detector performance for the node design described in the input file.

\section{Conclusions}

The work described in this paper has qualitatively shown that clutter has a negligable impact on neutron detection, while clutter has a measurable effect on photon detection. The effect of clutter is particularly dependent on the distance of the detector from the source, but it is also influenced by the type of shielding surrounding the source. These results justified the need for a photon clutter scaling algorithm, and such an algorithm was created that can 
be implemented into NINESIM.

Future work includes performing an uncertainty analysis on the ROC curve results in order to verify the choice of fitting functions and determine in what scenarios a scaling factor is unneccesary. Lastly, more scenarios must be simulated on MCNP in order to uncover all situations where clutter has a significant impact on detector performance.

\section{CONTRIBUTIONS MADE TO THE RESEARCH PROJECT}

My main contribution to the photon clutter work was selecting what cases should be run on MCNP, determining what fitting functions were needed for each of the data sets, and creating the photon clutter scaling algorithm based off the results. Additionally, I was able to do some work on impementing the algorithm into NINESIM near the end of my internship.

Most of the time I spent during this internship was creating a comprehensive test script, which will be used to verify that NINESIM functions correctly in the case that major modifications are made to the code. While in the process of making this script, I caught a bug in NINESIM that would cause errors in the generation of ROC curves that are not immediatly obvious. I also found a minor bug that caused an error in the path the cars took through the node. Both of these issues were fixed before the end of my internship.

\section{SKILLS AND KNOWLEDGE GAINED}

I came into this internship with very little programming experience. I have only taken one serious college-level coding class, with MATLAB being the language that was used. Creating the test script for NINESIM helped me become much more familiar with Python, as well as improve my coding skills in general. The nuclear engineering coursework at my university does not have a strong emphasis in programming, so my involvement in this project has helped me gain coding skills that I would not have had learned in my classes.

Performing the photon detection simulations on MCNP introduced me to the concept of ROC curves, which I was not aware of before. My familiarity with ROC curves could prove useful in future work that involves evaluating the performance of radiation detectors. 
Furthermore, running these simulations required that I use features of MCNP related to source definition and tallies that I have never used before.

I also learned quite a bit outside of work by attending lectures and tours throughout the summer. I attended a conference hosted by the Center for Strategic \& International Studies where I heard speakers discuss a number of issues such as arms control and deterrence. Lastly, my division (NEN) held a tour of the Device Assembly Facility at the Nevada National Security Site where I listened to staff discuss the work they do. I watched staff setup and perform a criticality experiment, and I also had the oppurtunity to hold a neptunium sphere and BeRP sphere with my own hands.

\section{IMPACT OF INTERNSHIP ON MY ACADEMIC/CAREER PLANNING}

During my freshman year I became a research assistant working on projects for researchers at the Pacific Northwest National Laboratory. This work sparked a desire to find out what working at the DOE national laboratories is like, and I also developed an interest in research related to national security. Because of this, my primary goal coming into this internship was finding out whether I wanted to pursue a career at the national laboratories, and, more generally, if I would like to continue on this path of doing research by attending graduate school. I would say that the ten weeks I spent at Los Alamos National Laboratory cemented my interest in pursuing a research career at the national laboratories.

\section{RELEVANCE OF RESEARCH TO THE MISSION OF DHS}

One of the core missions of the DHS is to "prevent terrorism and enhance security." The NINESIM project supports this mission by giving the DNDO a tool that can enhance the effectivenss of the office in preventing the illicit movement of radiological and nuclear materials within the United States. NINESIM can be used to optimize the design of an arbitrary GNDA node so that resources can be allocated efficiently in the construction of these nodes. This tool can also be used to test the theoretical capabilities of novel detector technologies that are in the early stages of research; this feature of NINESIM allows the DNDO to determine which projects show the most promise. 


\section{References}

[1] James Tutt and Gregg McKinney. Speed and Memory Improvement to MCNP6 DelayedGamma Line Treatment. Transactions of the American Nuclear Society, 116:949-952, 2017.

[2] Garrett McMath and Gregg McKinney. MCNP6 Study of Spatial Fidelity Required for ROC Curve Convergence. LANL Report: LA-UR-16-20255, ANS Annual Summer Meeting, New Orleans, LA, June 2016.

[3] Garrett McMath, Trevor Wilcox, and Gregg McKinney. How to Use the Receiver Operating Characteristic Tally Option in MCNP6. LANL Report: LA-UR-15-20195, Joint International Conference Mathematics and Computation, Supercomputing in Nuclear Applications (SNA) and the Monte Carlo (MC) Method, Nashville, TN,, April 2015.

[4] R.L. Heath. Scintillation Spectrometry: Gamma-Ray Spectrum Catalogue, volume 1, chapter 8, page 28. Idaho National Laboratory, 2nd edition, February 1997. 